ISBN 978-981-14-1684-2

Proceedings of 2019 the 9th International Workshop on Computer Science and Engineering

(WCSE 2019 SUMMER)

Hong Kong, 15-17 June, 2019, pp. 99-107

doi: $10.18178 /$ wcse.2019.06.015

\title{
Multi-Objective Optimization Recommendation Algorithm Based on Collaborative Filtering and Item Similarity
}

\author{
Chaosheng Zhao ${ }^{+}$ \\ School of Big Data \& Software Engineering, Chongqing University, China
}

\begin{abstract}
The traditional collaborative filtering recommendation algorithm generates recommendations for users by calculating the similarity between users or items, without considering other factors and data sparsity problem. The appearance of personalized tags can reflect the characteristics of items. Some algorithms try to optimize the recommendation results by setting weight coefficients of various factors. However, it is unreasonable to set the same weight coefficients for different users. To solve these problems, we propose a multi-objective optimization recommendation algorithm based on collaborative filtering and item similarity. First. The algorithm calculates the similarity between users by improved user-based collaborative filtering recommendation algorithm and generates a candidate recommendation set. Then, the algorithm uses the coupled object similarity measure method to calculate the similarity between items. Finally, we use NNIA algorithm to optimize the former two goals and generate the final recommendation results. Experimental results on the Movielens dataset shows that the proposed algorithm has good recommendation effect.
\end{abstract}

Keywords: collaborative filtering, item similarity, multi-objective optimization, NNIA.

\section{Introduction}

With the development of internet technology, information resources are increasing at the speed of geometric magnitude every day, which makes information overload problem increasingly prominent. In order to solve the problem that users have difficulty obtaining information, personalized recommendation algorithms come into being. At present, personalized recommendation algorithms have been applied to many places, such as e-commerce[1] and social networks[2][3]. Personalized recommendation algorithms mainly include content-based recommendation algorithm[4] and collaborative filtering recommendation algorithm[5]. The collaborative filtering recommendation algorithm is mainly divided into two categories: user-based collaborative filtering recommendation algorithm[6] and item-based collaborative filtering recommendation algorithm[7]. In the real world, there are tens of thousands of items, the items that users have evaluated are only a small part. Which leads to the data sparsity problem[8]. In addition, it is inaccurate to generate the recommendation results just by the predicted ratings.

To solve the above problems, we propose a multi-objective optimization recommendation algorithm based on collaborative filtering and item similarity. First, the algorithm calculates the similarity between users by improved user-based collaborative filtering recommendation algorithm and generates a candidate recommendation set. Then, the algorithm calculates the similarity between items by the coupled object similarity measure method[9]. Finally, we use the Non-dominated Neighbor Immune Algorithm (NNIA)[10] to simultaneously optimize the former two goals and generate the final recommendation results.

\section{Related Work}

\subsection{User-based collaborative filtering recommendation algorithm}

\footnotetext{
+ Corresponding author. Tel.: +8615023660490 .

E-mail address: $1145460677 @ q q . c o m$.
} 
The traditional user-based collaborative filtering recommendation algorithm discovers the user's preferences for items by user's historical behaviors. Then, the algorithm calculates the similarity between users according to the preferences. Finally, the algorithm generates the recommendation results according to the similarity between users. There are usually two methods for calculating the similarity between users: cosine similarity and correlation similarity[11][12].

After calculating the similarity between users, the algorithm will predict the item ratings by the kNearest Neighbor (KNN)[13] neighbor method. The target user's ratings of the items are predicted based on the real item ratings of the nearest neighbors. The formula is as follows:

$$
R(u, i)=\frac{\sum_{u \in N_{u}} \operatorname{Sim}\left(u, u^{\prime}\right) \cdot r_{u^{\prime}, i}}{\sum_{u^{\prime} \in N_{u}} \operatorname{Sim}\left(u, u^{\prime}\right)}
$$

where, $N_{u}$ represents the set of nearest neighbors, $r_{u^{\prime}, i}$ represents the real rating of the user $u^{\prime}$ for the item $i$.

\subsection{Coupled object similarity measure}

In the recommendation system, each item can be represented as a feature vector $\mathbf{O}=\left\{\mathrm{a}_{1}, \mathrm{a}_{2}, \ldots, \mathrm{a}_{\mathrm{m}}\right\}$, where each feature $a_{i}$ is a feature information. When the feature information is described by numerical data, the similarity between the items can be measured by the Euclidean distance and the Minkowski distance. If the feature information is described by the categorical data, the simple matching similarity using 1 and 0 to distinguish the similarity is not accurate. The coupled object similarity takes into account both Intra-coupled and Inter-coupled Attribute Value Similarities (IaAVS and IeAVS), which capture the attribute value frequency distribution and feature dependency aggregation with a high learning accuracy and relatively low complexity[14]. See the literature[9] for details.

\subsection{Multi-objective optimization algorithm}

There are many actual problems that involve simultaneously resolving several conflicting objectives, which are known as multi-objective optimization problems (MOPs)[10]. The multi-objective optimization problems(MOPs) can be described as:

$$
F(x)=\left(f_{1}(x), f_{2}(x), \ldots, f_{m}(x)\right) \text { s.t } x \in \Omega
$$

where $F(x)$ is the multi-objective optimization result. $f_{m}(x)$ is the target component.

In the minimization optimization problems, if and only if $\forall \mathrm{i} \in\{1,2, \ldots, \mathrm{m}\}, \mathrm{f}_{\mathrm{i}}(\mathrm{x}) \leq \mathrm{f}_{\mathrm{i}}(\mathrm{y})$, and $\exists$ $\mathrm{j} \in\{1,2, \ldots, \mathrm{m}\}, \mathrm{f}_{\mathrm{j}}(\mathrm{x})<\mathrm{f}_{\mathrm{j}}(\mathrm{y})$, which is called $\mathrm{x}$ dominates $\mathrm{y}$. If a solution is called the Pareto-optimal solution, it should not be dominated by other solutions[15]. For a multi-objective optimization problem, if the solution in a set is non-dominated with each other, the set is called Pareto-optimal solution set.

Evolutionary algorithms are a heuristic approach, the principle of which comes from the biological evolution of nature. The evolutionary algorithms can get the approximate Pareto-optimal solution of the problem in a single run. Because of that, evolutionary algorithms are very suitable for dealing with multiobjective optimization problems[16]. Many evolutionary algorithms have emerged in recent years. For example, MISA[17], NSGA-II[18], MOEA/D[19], NNIA[10], and so on.

\section{Multi-objective Optimization Recommendation Algorithm Based on Collaborative Filtering and Item Similarity}

\subsection{Algorithm design}

The proposed algorithm mainly includes three steps. The first step is to generate a candidate recommendation set by improved user-based collaborative filtering recommendation algorithm according to user information and user-item rating matrix. In this step, we use the user's history rating information to analyze the similarity between users and predict item ratings. Then, the ratings are sorted from high to low, and the first q items are selected as the candidate recommendation set. In the second step, we use the coupled object similarity measure method to calculate the similarity between items according to item information and the user-item tag information. Finally, we use the multi-objective optimization algorithm to simultaneously 
optimize the former two goals and generate the final recommendation results. The idea of the algorithm is shown in Figure 1.

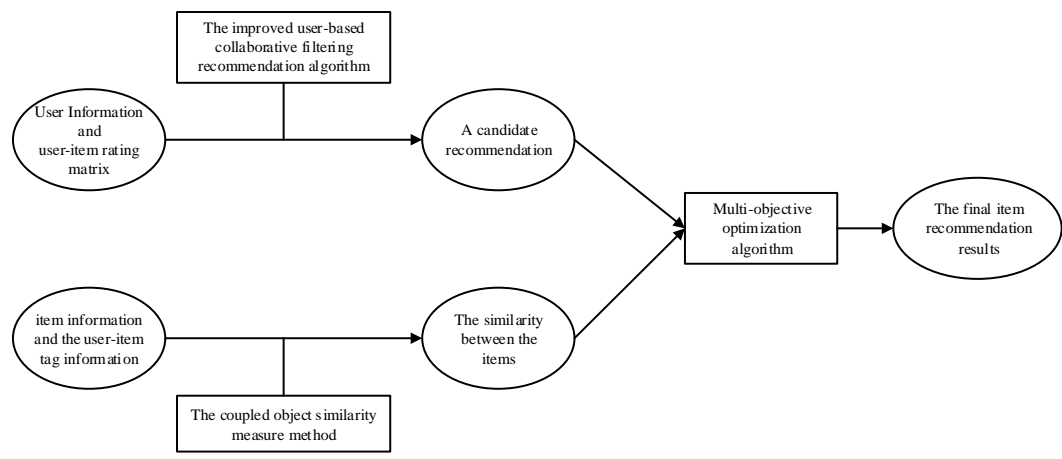

Fig. 1: multi-objective optimization recommendation algorithm based on collaborative filtering and item similarity.

\subsection{Improved user-based collaborative filtering recommendation algorithm}

The traditional user-based collaborative filtering recommendation algorithm needs to calculate all item ratings. which can increase the time complexity of the algorithm. In order to solve the data sparsity problem and reduce the time complexity of the algorithm, this paper proposes to calculate the similarity between users who have the common ratings of items. This greatly reduces the time complexity of the algorithm.

In order to calculate the similarity between users more accurately, this paper improves the similarity calculation method between users. It is proposed that the similarity between users includes both the similarity of the user's rating data and the similarity of the number of items that the users common rated. The formula is as follows:

$$
\operatorname{Sim}(x, y)=\operatorname{Sim}^{t}(x, y) \cdot \operatorname{Sim}^{c}(x, y)
$$

where $\operatorname{Sim}^{t}(x, y)$ represents the similarity of the user's rating data. $\operatorname{Sim}^{c}(x, y)$ represents the similarity of the number of items that the users common rated. The formulas are as follows:

$$
\operatorname{Sim}^{\mathrm{t}}(x, y)=\frac{\sum_{i \in I_{x, y}} r_{x, i} r_{y, i}}{\sqrt{\sum_{i \in I_{x}} r_{x, i}^{2}} \sqrt{\sum_{i \in I_{y}} r_{y, i}^{2}}}
$$

where $r_{x, i}$ represents the rating of user $x$ for item i, $r_{y, i}$ represents the rating of user $y$ for item $i, I_{x, y}$ represents the item set that user $\mathrm{x}$ and $\mathrm{y}$ rated, and $\mathrm{I}_{\mathrm{x}}$ and $\mathrm{I}_{\mathrm{y}}$ represent the item set the user $\mathrm{x}$ and $\mathrm{y}$ rated, respectively.

$$
\operatorname{Sim}^{c}(x, y)=\frac{|N(x) \cap N(y)|}{\sqrt{|N(x)||N(y)|}}
$$

where $\mathrm{N}(\mathrm{x})$ represents the item set of the user $\mathrm{x}$ rated, and $\mathrm{N}(\mathrm{y})$ represents the item set of the user y rated.

Besides, considering the user deviation, this paper makes an improvement on the formula (1):

$$
R(u, i)=\overline{r_{u}}+\frac{\sum_{u_{i \in N_{u}}} \operatorname{Sim}\left(u, u^{\prime}\right)\left(r_{u^{\prime}, i}-\overline{r_{u^{\prime}}}\right)}{\sum_{u^{\prime} \in N_{u}} \operatorname{Sim}\left(u, u^{\prime}\right)}
$$

where, $r_{u}$ and $r_{u^{\prime}}$ represent the average rating of all items of user $u$ and user $u^{\prime}$, respectively.

\subsection{Improvement of coupled object similarity measure method}

In this paper, we need to process the characteristics of the items and extract features of the numerical data from the item as many as possible. Then, we use the Jaccard similarity to calculate the similarity of each feature. The formula is as follows: 


$$
\operatorname{Sim}\left(a_{i, m}, a_{j, m}\right)=\frac{\left|a_{i, m} \cap a_{j, m}\right|}{\mathrm{W}^{*}\left|a_{i, m} \cup a_{j, m}\right|}
$$

where $a_{i, m}$ and $a_{j, m}$ represent the $m$-th feature of item $i$ and $j$, respectively. Then, the algorithm sums all the similarities to get the final similarity.

With the advent of tag information, users can set tag information for items that they are interested in. The tag information provides more versatile features of the items. In this paper, the tag information is formed into a vector in the n-dimensional space vector $T=\left\{t_{1}, t_{2}, \ldots, t_{1}\right\}$. The size of the angle between the two vectors represents the similarity between items. The formula is as follows:

$$
\operatorname{Sim}(\mathrm{i}, j)=\cos \left(\mathbf{T}_{i}, \mathbf{T}_{j}\right)=\frac{\mathbf{T}_{i} \cdot \mathbf{T}_{j}}{\left|\mathbf{T}_{i} \| \mathbf{T}_{j}\right|}
$$

\subsection{Multi-objective optimization algorithm}

After generating the candidate recommendation set and the similarity of the items, we use the NNIA algorithm to optimize the targets because it proves to be an effective algorithm for solving multi-objective optimization problems. NNIA algorithm has effectively solved the recognition of network structures[20] and image segmentation problems[21]. In order to satisfy the requirements of the recommendation algorithm, we need to improve the genetic operation of the NNIA algorithm.

(1) The flow of NNIA algorithm

Input: maximum number of generation gmax, maximum size of dominant population NM, maximum size of active population NA, clone population size CS.

Step1 Initialization: Initialize the antibody population $\mathrm{B}_{0}$ randomly, set the non-dominant antibody population $\mathrm{D}_{0}=\varphi$, the active antibody population $\mathrm{A}_{0}=\varphi$, the clone antibody population $\mathrm{C}_{0}=\varphi$, and the calculator $\mathrm{t}=0$.

Step2 Update the dominant antibody population: select the dominant antibody in $\mathrm{B}_{\mathrm{t}}$ to form the nondominant antibody population $\mathrm{D}_{\mathrm{t}+1}$;

Step3 Terminate the judgment: If the terminated condition is satisfied, the output $D_{t+1}$ is used as the algorithm solution result, and the algorithm ends; otherwise, $\mathrm{t}=\mathrm{t}+1$.

Step4 Non-dominated neighborhood selection: Select the dominant antibody with a large crowding distance to form the active antibody population $\mathrm{A}_{\mathrm{t}}$.

Step5 Proportional cloning: Perform adaptive proportional cloning on $A_{t}$ to obtain a cloned antibody population $\mathrm{C}_{\mathrm{t}}$ with a clone size of $\mathrm{CS}$.

Step6 Generate new antibodies: perform crossover operation and mutation operation on $\mathrm{C}_{\mathrm{t}}$ to obtain $\mathrm{S}_{\mathrm{t}}$.

Step7 Update the antibody population: Obtain the antibody population $\mathrm{B}_{\mathrm{t}}=\mathrm{D}_{\mathrm{t}} \cup \mathrm{S}_{\mathrm{t}}$, and transfer to Step2.

(2) Initialization and genetic operation of the NNIA algorithm

In this paper, the initial population is randomly generated by binary coding, the antibody $X=\left\{x_{1}, x_{2}, \ldots, x_{q}\right\}$ of the initial population should satisfy the formula (9).

$$
\sum_{i=1}^{q} \mathrm{x}_{i}=N
$$

where $\mathrm{q}$ is the length of the antibody. $\mathrm{N}$ is the size of the final item recommendation set.

Genetic operation is the most important part of the NNIA algorithm. Genetic operation mainly includes crossover operation and mutation operation. In the recommendation list, a position can only appear once, so the genetic operation is improved to ensure which can adapt to the recommendation algorithm.

Crossover operation affects the two parent antibodies, and generates two children antibodies. In order to satisfy the requirement of formula (9), the standard single-point crossover operation is improved. It is assumed that $\mathrm{p}_{1}$ and $\mathrm{p}_{2}$ are two parent antibodies, and $\mathrm{c}_{1}$ and $\mathrm{c}_{2}$ are two daughter antibodies produced by the 
parent antibodies. The crossover process is as follows: In the first step, the algorithm initializes all genes of the children antibodies $c_{1}$ and $c_{2}$ to 0 . In the second step, if the value of the same position of $p_{1}$ and $p_{2}$ is 1 , then it should be set to 0 , and the same position of $c_{1}$ and $c_{2}$ is set to 1 . Assuming that $s$ is the position that satisfies the requirement, set $\mathrm{d}=\mathrm{q}-\mathrm{s}$. In the third step, let $\mathrm{cp}$ be a random integer of the interval [1, d-1]. For $\mathrm{p}_{1}$ : Copy the gene value before $\mathrm{cp}$ to the corresponding position of $\mathrm{c}_{1}$. At the same time, the remaining gene values are copied to the corresponding positions of $c_{2}$. A similar operation is performed for $\mathrm{p}_{2}$. The crossover operation is shown in Figure 2.

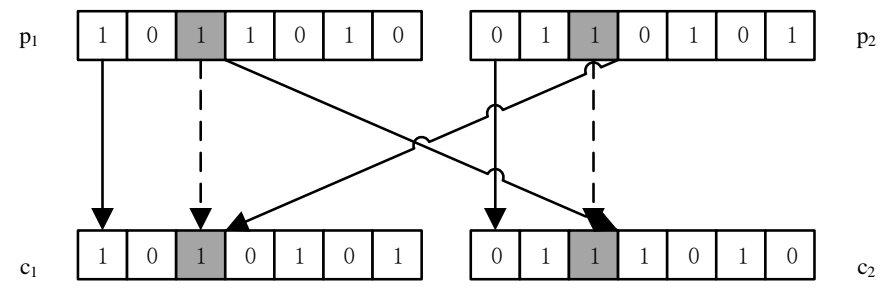

Fig. 2: An example for crossover operation. Assuming that $\mathrm{q}=7, \mathrm{~N}=4, \mathrm{~s}=3$ and $\mathrm{cp}=1$.

Mutation operation occurs randomly in each antibody. In this paper, two different integers of the interval $[1, \mathrm{~m}]$ are randomly generated, and then the symmetric rotation between the two points is performed according to a certain probability. The mutation operation is shown in Figure 3.
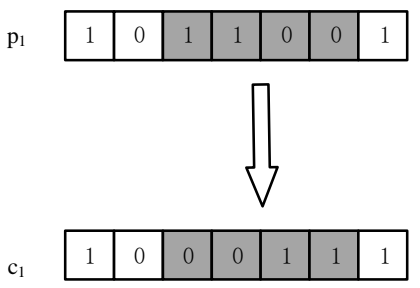

Fig. 3: An example for Mutation operation. Assuming that two different integers are 3 and 6.

(3) objective function

In some literatures, some scholars use the weight coefficients to adjust the influence of various factors to generate the final recommendation results. However, it is obviously unreasonable to use the same weight coefficients for all users. Because different users have different preferences for different factors. This paper proposes to optimize the user historical preferences and similarity between items simultaneously, which is different from the various algorithms proposed in the previous literatures. The objective functions of the algorithm are as follows:

$$
\begin{cases}\max & \mathrm{g}\left(\mathrm{i}, I_{u}\right) \\ \max & \mathrm{s}\left(\mathrm{i}, I_{u}\right)\end{cases}
$$

where, $g\left(i, I_{u}\right)$ is an objective function that reflects the similarity between items. $\mathrm{i}$ is the final item recommendation set. $I_{u}$ is the set of rated items of the target user $u$. The formula is as follows:

$$
\mathrm{g}\left(\mathrm{i}, I_{u}\right)=\sum_{\mathrm{i} \in L_{u}, j \in I_{u}} \operatorname{Sim}(i, j)
$$

where, $L_{u}$ is the final item recommendation set. $\operatorname{Sim}(x, y)$ is the similarity between the items $i$ and $j$, the calculation formula is as shown in the formula (12):

$$
\operatorname{Sim}(\mathbf{i}, j)=\cos (\mathbf{i}, \mathbf{j})=\frac{\sum_{u \in U_{\mathrm{i}, j}} r_{u, i} r_{u, j}}{\sqrt{\sum_{u \in U_{i}} r_{u, i}^{2}} \sqrt{\sum_{u \in U_{j}} r_{u, j}^{2}}}
$$

where $U_{i, j}$ is a set of users who have common ratings for items $i$ and $j$, and $U_{i}$ and $U_{j}$ represent a set of users who rated item $i$ and $j$, respectively. 
$\mathrm{s}\left(\mathrm{i}, \mathrm{I}_{\mathrm{u}}\right)$ is an objective function that reflects the user's preferences for new items. In this paper, we consider the similarity of the coupled object of the item features and the similarity of the coupled object based on the user-item tag information to obtain the user's preferences for the new items. The calculation formula is as follows:

$$
\mathrm{s}\left(\mathrm{i}, I_{u}\right)=\operatorname{Sim}(\mathrm{i}, \mathrm{j}) \sum_{m=1}^{w} \operatorname{Sim}\left(\mathrm{a}_{i, m}, \mathrm{a}_{j, m}\right)
$$

\section{Experiments and Analysis}

\subsection{Dataset}

The experimental data is the Movielens dataset that is opened by the GroupLens team. There are many characteristic information and ratings in the dataset. In addition, the dataset also includes user-tag-movie data information. In order to perform the experiments, we do a preliminary screening of the dataset information. At last, we use $80 \%$ of the data as the training set and the remaining $20 \%$ as the test set.

\subsection{Evaluation indicators}

The final output of the algorithm proposed in this paper is the item recommendation set for the target user. In order to evaluate the recommendation effect of the proposed algorithm, we use the accuracy, the recall rate and average absolute error MAE as the evaluation indicators. The accuracy is the proportion of the items that the user likes in the recommendation list, the calculation is formula (14). The recall rate is how many items the user likes in the test set appear in the recommendation list, the calculation is formula (15). As one of the commonly used evaluation Indicators in the recommendation algorithm, MAE measures the accuracy of the prediction by calculating the average deviation between the user's predicted ratings and the actual ratings. The calculation is as shown in formula (16).

$$
\begin{gathered}
\operatorname{accuracy}(U)=\frac{1}{|U|} \sum_{u \in U} \frac{L_{u} \cap B_{u}}{\left|L_{u}\right|} \\
\operatorname{recall}(U)=\frac{1}{|U|} \sum_{u \in U} \frac{L_{u} \cap B_{u}}{\left|B_{u}\right|}
\end{gathered}
$$

where $\mathrm{U}$ is the set of users in the test data, and $\mathrm{L}_{\mathrm{u}}$ is the recommendation item set. $\mathrm{B}_{\mathrm{u}}$ is the set of items that the user $u$ likes in the test set.

$$
M A E=\frac{\sum_{(u, j) \in \Omega^{\text {test }}}\left|R(u, j)-\mathrm{r}_{u, j}\right|}{\left|\Omega^{\text {test }}\right|}
$$

where $\Omega^{\text {test }}$ is a set of test data.

Table 1: Parameters setting

\begin{tabular}{cc}
\hline Parameters & Values \\
\hline q & 100 \\
gmax & 200 \\
NA & 20 \\
CS & 100 \\
NM & 40 \\
pc & 0.8 \\
pm & 0.1 \\
\hline
\end{tabular}

\subsection{Analysis of experimental results}


This paper proposes a multi-objective optimization recommendation algorithm based on collaborative filtering and item similarity. There are many parameters in the algorithm. Refer to the parameter settings in Literature[15]. The values of parameters are shown in Table 1.

(1) Experiments for the nearest neighbor $\mathrm{K}$

We can find that the quality of the candidate recommendation set directly affects the recommendation effect of the algorithm. The candidate recommendation set is determined by the nearest neighbors of the target user. Therefore, it is necessary to experiment with the selection of the nearest neighbor $\mathrm{k}$. In this paper, the value of the nearest neighbors $\mathrm{k}$ is set to increase from 10 to 70 , and the value of MAE is used as an experimental evaluation indicator. The experimental results are shown in Figure 4.

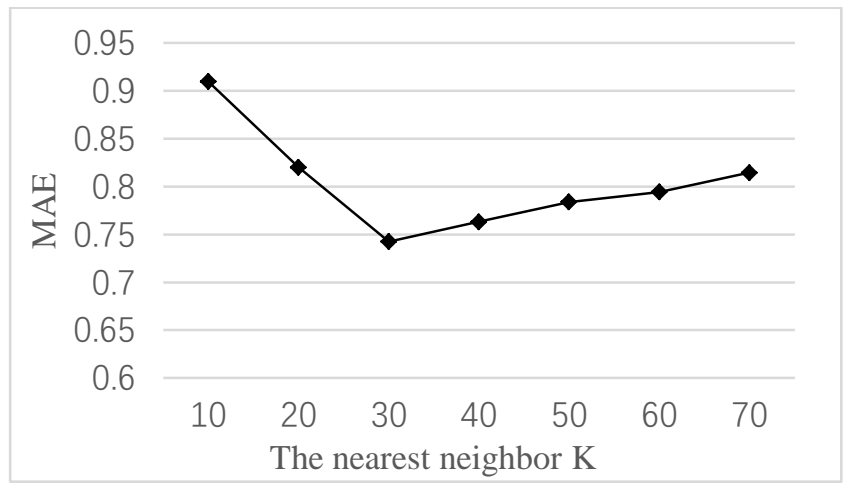

Fig. 4: Comparison of MAE under different values of nearest neighbors.

It can be seen from Figure 4 that as the value of the nearest neighbor increases, the value of MAE decreases first, and the effect of the candidate recommendation set gradually becomes better. When the $\mathrm{k}$ is 30, the MAE value is the smallest, and the effect of the candidate recommendation set is the best. After that, as the value of $\mathrm{k}$ increases, the MAE value gradually increases, and the effect of the candidate recommendation set deteriorates. Therefore, this paper considers that when the nearest neighbors $\mathrm{k}$ is taken as 30, the candidate recommendation set generated by the algorithm achieves the best effect.

(2) Comparison of this Algorithm with Other Algorithms

In order to evaluate the quality of the proposed algorithm in this paper, the algorithm is compared with the traditional user-based recommendation algorithm (User-CF), the hybrid collaborative filtering recommendation algorithm (Hybrid-CF)[22] and NSGA-II. The value of the nearest neighbor $\mathrm{k}$ is taken as 30. At the same time, in order to verify the effect of the number $\mathrm{N}$ of the final recommendation set on the recommendation effect, we set the value of $\mathrm{N}$ from 10 to 50 , and the increment value is 10 each time. We analyze the experimental results from three aspects: the accuracy, the recall rate and MAE.

Table 2: the accuracy of different algorithms

\begin{tabular}{ccccc}
\hline $\mathrm{N}$ & User-CF & Hybrid-CF & NSGA- II & This algorithm \\
\hline 10 & 0.0606 & 0.0742 & 0.1512 & 0.1424 \\
20 & 0.0514 & 0.0683 & 0.1284 & 0.1089 \\
30 & 0.0501 & 0.0610 & 0.1018 & 0.0824 \\
40 & 0.0505 & 0.0563 & 0.0724 & 0.0749 \\
50 & 0.0462 & 0.0497 & 0.0657 & 0.0671 \\
\hline \multicolumn{5}{c}{ Table 3: The recall rate of different algorithms } \\
\hline 10 & User-CF & Hybrid-CF & NSGA- II & This algorithm \\
20 & 0.0068 & 0.0084 & 0.1318 & 0.1527 \\
30 & 0.0149 & 0.0193 & 0.1684 & 0.1772 \\
40 & 0.0227 & 0.0287 & 0.1952 & 0.2023 \\
50 & 0.0307 & 0.0361 & 0.2307 & 0.2396 \\
\hline
\end{tabular}


It can be found from Table 2 and Table 3 that the proposed algorithm is better than the User-CF algorithm and the Hybrid-CF algorithm in terms of the accuracy and the recall rate. At the same time, it is found that the algorithm of this paper has no significant difference in accuracy and recall rate compared with NSGA-II algorithm. This is mainly because both of them optimize multiple targets at the same time to get the better recommendation results. Besides, it can be found that the recommendation effect become better and better, as the number of the final recommendation set increases.

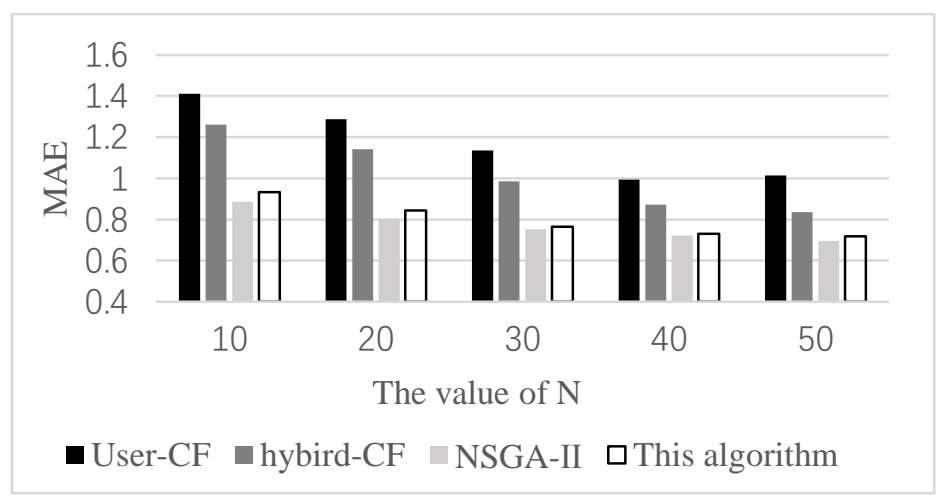

Fig. 5: Comparison of MAE under different algorithms

As we can see from Figure 5, the MAE value of the proposed algorithm is smaller than the MAE value of the User-CF algorithm and the Hybrid-CF algorithm. the predicted ratings of the algorithm are closer to the actual ratings than those two algorithms. The recommendation results are more accurate. At the same time, it is also found that the MAE value of the algorithm in this paper is bigger, compared with the MAE value of NSGA-II. This indicates the proposed algorithm in this paper still has some shortcomings. It mainly because we just think about the ratings and the characteristics of items.

\section{Conclusions}

This paper proposes a multi-objective optimization recommendation algorithm based on collaborative filtering and item similarity. On the one hand, the algorithm considers the user's historical preferences and the similarity between items, then recommends the items for users from various factors. On the other hand, the algorithm uses NNIA algorithm for multi-objective optimization, which avoids the use of parameter tuning. compared with some existing recommendation algorithms, the proposed algorithm has better results in terms of the accuracy, the recall rate and MAE. However, Since the algorithm generates the candidate recommendation set just according to user's historical preferences, this affects the accuracy of the candidate recommendation set to some extent. In the future research, we will pay more attention to the selection of candidate recommendation sets and multi-objective optimization. We will also optimize the algorithm from more factors so that the algorithm can generate the better recommendation results.

\section{References}

[1] Sun, Li \& Pan, ChengEn. (2014). Personalized recommendation system based on data mining to E-commerce. WIT Transactions on Information and Communication Technologies. 54. 91-98.

[2] Gao H, Tang J, Liu H. Personalized location recommendation on location-based social networks[J]. 2014.

[3] Yu, Fei. (2017). Personalized POI Groups Recommendation in Location-Based Social Networks. Lecture Notes in Computer Science.

[4] Shu J, Shen X, Liu H, et al. A content-based recommendation algorithm for learning resources[J]. Multimedia Systems, 2017(1):1-11.

[5] Zhenhai Y, Yonghao F, Yikun Z, et al. The Research of Modified Collaborative Filtering Recommendation Algorithm[C]// International Conference on Information Technology in Medicine \& Education. IEEE, 2016.

[6] Ge F. A User-Based Collaborative Filtering Recommendation Algorithm Based on Folksonomy Smoothing[J]. Communications in Computer and Information Science, 2011, 202.

[7] Su, Ping \& Ye, HongWu. (2009). An Item Based Collaborative Filtering Recommendation Algorithm Using 
Rough Set Prediction. 308-311.

[8] Adomavicius G, Tuzhilin A. Toward the Next Generation of Recommender Systems: a Survey of the State-of-theart and Possible Extensions[J]. IEEE Trans on Knowledge and Data Engineering, 2005, 17(6): 734-749.

[9] Wang, Can \& Cao, Longbing \& Wang, Mingchun \& Li, Jinjiu \& Wei, Wei \& Ou, Yuming. (2011). Coupled nominal similarity in unsupervised learning. 973-978.

[10] M. Gong, L. Jiao, H. Du, L. Bo, Multiobjective immune algorithm with nondominated neighbor-based selection, Evol. Comput. 16 (2) (2008) 225-255.

[11] Breese, John S, Heckerman, David, Kadie, Carl. Empirical analysis of predictive algorithms for collaborative filtering[J]. Uncertainty in Artificial Intelligence, 2013, 98(7):43-52.

[12] Sarwar B, Karypis G, Konstan J, et al. Item-based collaborative filtering recommendation algorithms[C]// International Conference on World Wide Web. ACM, 2001:285-295.

[13] Li, Gang \& Zhang, Jingjing. (2018). Music personalized recommendation system based on improved KNN algorithm.

[14] Melville P, Mooney R J, Nagarajan R. Content-boosted collaborative filtering for improved recommendations[C]// Eighteenth national conference on Artificial intelligence. American Association for Artificial Intelligence, 2002.

[15] Bingrui Geng, Licheng Jiao, Maoguo Gong, Lingling Li, Yue Wu. A two-step personalized location recommendation based on multi-objective immune algorithm[J]. Information Sciences, 2019, 475: 161-181.

[16] Zhou, Aimin \& Qu, Bo-Yang \& Li, Hui \& Zhao, Shi-Zheng \& Suganthan, Ponnuthurai \& Zhang, Qingfu. (2011). Multiobjective evolutionary algorithms: A survey of the state of the art. Swarm and Evolutionary Computation. 1. $32-49$.

[17] Coello C A C, Cortes N C. An Approach to Solve Multiobjective Optimization Problems Based on an Artificial Immune System[C]// International Conference on Artificial Immune Systems. 2002.212-221.

[18] Deb K, Pratap A, Agarwal S, et al. A fast and elitist multiobjective genetic algorithm: NSGA-II[J]. IEEE Transactions on Evolutionary Computation, 2002, 6(2):182-197.

[19] Zhang Q, Li H. MOEA/D: A Multiobjective Evolutionary Algorithm Based on Decomposition[J]. IEEE Transactions on Evolutionary Computation, 2007, 11(6):712-731.

[20] Gong M, Chen X, Ma L, et al. Identification of multi-resolution network structures with multi-objective immune algorithm[J]. Applied Soft Computing, 2013, 13(4):1705-1717.

[21] Yang D, Jiao L, Gong M, et al. Artificial immune multi-objective SAR image segmentation with fused complementary features[J]. Information Sciences An International Journal, 2011, 181(13):2797-2812.

[22] Ji H, Li J, Ren C, et al. Hybrid collaborative filtering model for improved recommendation[C]// IEEE International Conference on Service Operations \& Logistics. IEEE, 2013. 